\title{
THORAX
}

Editorials

\section{Use of near fatal asthma for investigating asthma deaths}

It is an understatement to observe that there has been increasing concern about mortality from asthma in recent decades and considerable controversy regarding the appropriate methods for investigating the causes of asthma mortality. Until recently such investigations were based on descriptive surveys in which the characteristics of the asthmatic subject and the circumstances of death were documented following interviews with relatives, close friends or attending medical staff, together with a review of medical records. Difficulties with documenting the actual events before death (which were often unwitnessed), however, and the lack of a control group against which comparisons could be made, limited the usefulness of these surveys and often led to conflicting conclusions.

In 1986 Rea et al $^{1}$ undertook a small case control study of asthma mortality using both hospital and community based controls. This pioneering study was a significant advance on the simpler case survey approach and allowed risk factors for death from asthma to be quantified.

Case control methodology was subsequently employed to specifically investigate the possible role of prescribed drug treatment in asthma mortality. ${ }^{2}$ In this way a series of case control studies from New Zealand identified that the use of the high dose preparation fenoterol was most likely to be the major factor in the recent epidemic of asthma deaths in that country. ${ }^{3}$ As the majority of deaths from asthma occur outside hospital it was generally necessary to confine these case control studies to the subgroup of patients with a recent hospital admission, so that information on regular prescribed medication and markers of chronic asthma severity could be obtained from routine hospital records. However, this approach incorporated fewer than half of all asthma deaths and was designed to address the specific hypothesis concerning the role of fenoterol in the second New Zealand mortality epidemic. Such an approach is of less value in addressing other causes of asthma mortality for which information must be collected by personal interviews.

It is thus appealing to consider the hypothesis that fatal and near fatal attacks of asthma may have common causes, and that studying non-fatal asthma attacks (as well as being of value in itself) may provide useful information on the factors associated with fatal asthma attacks. Two papers in this issue of Thorax address the use of near fatal asthma for investigating asthma deaths.

Campbell $e t a l^{\beta}$ investigated the levels of agreement between information obtained directly from cases of near fatal asthma attacks with those obtained independently from close acquaintances, almost all of whom were family members living in the same household. They found very high levels of agreement for recognised markers of chronic asthma severity (such as a hospital admission in the last 12 months or an intensive care unit admission), ${ }^{4}$ utilisation of medical services, the use of prescribed $\beta$ agonists (although there was less agreement for other asthma medications such as oral xanthines), and psychiatric features. These findings are generally encouraging in that they indicate that information relating to these risk factors and clinical characteristics obtained from close acquaintances has reasonable reliability in studies of near fatal asthma attacks and therefore such information may also be reliable for persons dying from asthma. In contrast, information on features of the long term asthma history such as sleep disturbance and the frequency of, or trends in, symptoms in previous years was not reliable. The reliability of the reported circumstances associated with a near fatal episode was not examined. Thus, while it would seem appropriate to continue to examine deaths from asthma using clinical information that has been recorded before the fatal attack, the findings of Campbell et al suggest that interviews with acquaintances may provide additional useful data.

Richards et $a l^{5}$ have compared the demographic characteristics of patients suffering fatal asthma attacks throughout New Zealand during 1980-6 with those experiencing severe life threatening asthma attacks in the Auckland region during 1981-7. The information available for the study was limited, but the findings once again confirm the importance of a previous life threatening attack of asthma as a marker of subsequent risk of death from asthma. ${ }^{1}$ About one quarter of the subjects who died in Auckland during the period of the study had a previous admission to an intensive care unit with asthma. This suggests that asthmatic subjects who experience a life threatening attack are a group in whom intensive medical treatment and follow up should be arranged. The value of such an approach has been suggested by the South Australian experience in which an intensive specialist based medical programme for patients with near fatal asthma led to a significant reduction in morbidity. ${ }^{6}$ Furthermore, during the mean follow up period of two and a half years after a near fatal attack there were no deaths in their 45 patients-an experience which contrasts with that reported in the study from Auckland where a $7 \cdot 5 \%$ mortality rate was observed in the two years after a near fatal attack. ${ }^{5}$

In addition to its clinical usefulness in identifying high risk patients, there are further uses for information on near fatal asthma attacks. Firstly, monitoring of time trends in near fatal asthma may complement the monitoring of time trends in fatal asthma. Compared with deaths from asthma which are relatively rare and in which reporting and confirmation is relatively slow, near fatal attacks (at least those involving admissions to an intensive care unit) are more common and can be identified relatively easily. Thus, monitoring increases in near fatal attacks in a country or region may serve as an "early warning system" when there is concern about the possibility of an increase in mortality. Secondly, case control studies of near fatal asthma attacks may serve as a useful complement to case control studies of fatal attacks, as with the recent Saskatchewan study. ${ }^{7}$ Thirdly, the study of near fatal attacks has some practical advantages in that detailed information of the circumstances of the severe attack can sometimes be obtained, which is usually not available in fatal asthma attacks. Furthermore, if hospital admission controls are used it is possible to interview both cases and controls and to obtain information from hospital records using similar procedures. 
However, there are also reasons for caution in the use of information on near fatal asthma attacks. Firstly, it should be emphasised that some factors may increase the risk of both fatal and near fatal attacks, whereas other factors may solely affect whether a particular attack is fatal. For example, if there are changes in prehospital care which reduce delays in receiving urgent medical treatment, there may be an increase in hospital and intensive care unit admissions and a corresponding decrease in asthma deaths, as Richards et al proposed as an explanation for the time trends presented. ${ }^{5}$ Secondly, obtaining appropriate controls may be difficult in case control studies of near fatal attacks. Previous studies have shown that asthma deaths and asthma hospital admissions are very similar with respect to recognised markers of chronic asthma severity. ${ }^{1}$ Thus, an adequate match for asthma severity can be obtained in case control studies in which asthma deaths are compared with hospital admission controls. However, the same may not be true in case control studies of near fatal attacks as the subgroup of asthmatic patients experiencing near fatal attacks to some extent represents a population of "survivors" who have frequent hospital admissions and high usage of asthma drugs; these factors may be responsible for the survival of this subgroup rather than for the occurrence of the severe attacks themselves.

These considerations indicate that, although there are some practical advantages in studying near fatal asthma attacks, there may well be greater problems of interpretation of the findings than in studies of fatal asthma. Nevertheless, when interpreted cautiously, studies of near fatal attacks may provide a useful complement to studies of fatal attacks in monitoring the time trends in severe asthma, and in identifying the causes of those changes which occur.

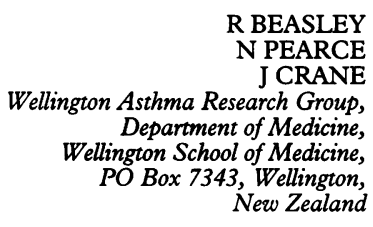

Reprint requests to: Professor R Beasley

1 Rea HH, Scragg R, Jackson R, Beaglehole R, Fenwick J, Sutherland D A case-control study of deaths from asthma. Thorax 1986;41:833-9.

2 Pearce NE, Crane J, Burgess C, Jackson R, Beasley R. Beta agonists and asthma mortality: déjà vu. Clin Exp Allergy 1991;21:401-10.

3 Campbell DA, McLennan G, Coates JR, Frith PA, Gluyas PA, Latimer $\mathrm{KM}$, et al. Near fatal asthma attacks: the reliability of descriptive information collected from close acquaintances. Thorax 1993;48:1099-104.

4 Crane J, Pearce NE, Burgess C, Woodman K, Robson B, Beasley R Markers of risk of asthma death or readmission in the 12 months following a hospital admission for asthma. Int 7 Epidemiol 1992;21:737-44.

5 Richards G, Kolbe J, Fenwick J, Rea H. Demographic characteristics of patients with severe life threatening asthma: comparison with asthma deaths. Thorax 1993;48:1105-9.

6 Ruffin RE, Latimer KM, Schembri DA. Longitudinal study of near fatal asthma. Chest 1991;99:77-83.

7 Spitzer WHO, Suissa S, Ernst P, Horwitz RI, Habbick B, Cockcroft D, et al. The use of beta-agonists and the risk of death and near death from asthma. $N$ Engl f Med 1992;326:501-6. 